\title{
Comment on "Obliquely propagating large amplitude solitary waves in charge neutral plasmas" by F. Verheest, Nonlin. Processes Geophys., 14, 49-57, 2007
}

\author{
J. F. McKenzie ${ }^{1}$, E. Dubinin ${ }^{2}$, and K. Sauer ${ }^{2}$ \\ ${ }^{1}$ Schools of Pure and Applied Physics and Mathematical Sciences, University of KwaZulu-Natal, Durban, South Africa \\ ${ }^{2}$ Max-Planck Institut für Sonnensystemforschung, Katlenburg-Lindau, Germany
}

Received: 15 May 2007 - Revised: 10 August 2007 - Accepted: 17 August 2007 - Published: 27 August 2007

In a recent paper on nonlinear stationary waves in a magnetized plasma, Verheest (2007) imposed the constraint of "strict" charge neutrality by which is meant that simultaneously with $n_{i}=n_{e}$ the longitudinal electric field $E_{x}$ is strictly zero. Armed with this constraint Verheest (2007) goes on to show that nonlinear whistler waves, oscillitons and obliquely propagating Hall-MHD solitons do not exist. This is a case of throwing the baby out with the bathwater.

There are circumstances in which quasi-charge neutrality holds with $n_{i} \approx n_{e}$ but $\operatorname{div} \boldsymbol{E} \neq 0$. The nonrelativistic whistler wave is one such circumstance. In this case we may write Poisson's equation in the form

$\frac{1}{\omega_{p e}^{2}} \frac{d}{d x}\left(\frac{e E_{x}}{m_{e}}\right)=\frac{\delta n}{n}$

in which $\omega_{p e}$ is the electron plasma frequency and $\delta n / n$ is the ratio of excess of ions over electrons to the plasma density. The whistler oscilliton propagates at approximately $V_{A e} / 2$ speed (where $V_{A e}$ is the Alfven speed based on the electron mass density) with a characteristic length scale $V_{A e} / \Omega_{e}$ (where $\Omega_{e}$ is the electron gyrofrequency) and an electron acceleration $V_{A e} \Omega_{e} / 4$ (see e.g. Sauer et al., 2002; Dubinin et al., 2003; McKenzie et al., 2004; Mace et al., 2007). Equation (1) then shows that

$$
\frac{\delta n}{n} \sim \frac{\Omega_{e}^{2}}{4 \omega_{p e}^{2}}=\frac{V_{A e}^{2}}{4 c^{2}}
$$

Hence quasi-charge neutrality $n_{i} \approx n_{e}$ and $E_{x} \neq 0$ is a good approximation if the wave speed $V_{A e} / 2$ is not relativistic. According to Chen (1984) "the novice finds this property difficult to understand". On the other hand, if $V_{A e}$ is of the order of $c$ then we must not only use Poisson's equation but also the equations of motion must be made relativistic as discussed by McKenzie et al. (2005).

Correspondence to: E. Dubinin

(dubinin@mps.mpg.de)
A similar argument can be made for Hall-MHD solitons in which the acceleration of the protons is $0\left(V_{A} \Omega_{p}\right)$, the length scale is $V_{A} / \Omega_{p}$ yielding the fractional charge separation $\delta n / n \sim \Omega_{p}^{2} / \omega_{p i}^{2}=V_{A}^{2} / c^{2}$, where $V_{A}$ and $\Omega_{p}$ are the Alfven speed based on the proton mass density and the proton gyrofrequency, respectively, demanding that $\delta n / n$ is relativistically small and $\operatorname{div} \boldsymbol{E} \neq 0$. This is the reason why the standard Hall-MHD equations do not require Poisson's equation and do not impose any constraint on $\operatorname{div} \boldsymbol{E}$. The electric fields are derived from Faraday's law and the equations of the motion together with the condition $\operatorname{div} \boldsymbol{j}=0$. Poisson's equation provides us only with an estimate of the charge separation ratio $\delta n / n$ as was shown above.

In the classic nonlinear wave propagating transverse to the magnetic field (Adlam-Allen, 1958; Sagdeev, 1966) the circumstances are not quasi-charge neutral. In fact, such an assumption would violate conservation of longitudinal momentum requiring a balance between dynamic pressure and magnetic pressure. It is interesting that in this case the electric stresses are again relativistically small compared with magnetic stresses (pressure) but Poisson's equation is not required even with $n_{i} \neq n_{e}$ (McKenzie et al., 2001).

By setting $E_{x}=0$ simultaneously with $n_{i}=n_{e}\left(u_{i x}=u_{e x}\right)$ Verheest (2007) has taken a wrong turning and got a paradoxical result even for the linear (evanescent) waves. In conclusion, note that a question of using Poisson's equation versus the quasineutrality condition is not a new one. It was discussed and clarified 40 years ago in the classical review paper by Braginskii (1965).

Edited by: J. Büchner

Reviewed by: M. Leubner and another anonymous referee 


\section{References}

Adlam, J. H. and Allen, E.: The structure of strong collision-free hydromagnetic waves, Phil. Mag., 3, 448, 1958.

Braginskii, S. I.: Transport processes in a plasma, in: Reviews of Plasma Physics, v. 1, edited by: Leontovich, M. A., Consultants Bureau, New-York, 1965.

Chen, F. F.: in: Plasma Physics. Controlled Fusion, p. 77, Plenum Press, New-York, 1984.

Dubinin, E., Sauer, K., and McKenzie, J. F.: Nonlinear stationary whistler waves and whistler solitons (oscillitons). Exact solutions, J. Plasma Phys., 69, 305-330, 2003.

Mace, R. L., McKenzie, J. F., and Webb, G. M.: Conservation laws for steady flow and solitons in multi-fluid plasma revisited,, Phys. Plasmas, 14, 012310-1, 2007.
McKenzie, J. F., Dubinin, E., and Sauer, K.: Nonlinear waves propagating transverse to the magnetic field, J. Plasma Phys., 65, 213-233, 2001.

McKenzie, J. F., Dubinin, E., Sauer, K., and Doyle, T. B.: The application of the constants of motion to nonlinear stationary waves in complex plasmas: a unified fluid dynamic viewpoint, J. Plasma Phys., 70, 431-462, 2004.

Sagdeev, R. Z.: Cooperative phenomena and shock waves in collisionless plasmas, in: Reviews of Plasma Physics, v. 4, edited by: Leontovich, M. A., Consultants Bureau, New-York, p. 23, 1966.

Sauer, K., Dubinin, E., and McKenzie, J. F.: Wave emission by whistler oscillitons: Application to "coherent lion roars", Geophys. Res. Lett., 29, 2226, doi:10.1029/2002GL015771, 2002.

Verheest, F.: Obliquely propagating large amplitude solitary waves in charge neutral plasmas, Nonlin. Processes Geophys., 14, 49-57, 2007, http://www.nonlin-processesgeophys.net/14/49/2007/. 